\title{
Correction to: the IL1RN Mutation Creating the Most-Upstream Premature Stop Codon Is Hypomorphic because of a Reinitiation of Translation
}

\author{
Kunihiko Moriya ${ }^{1,2,3}$ - Saori Kadowaki ${ }^{4} \cdot$ Tomohiro Nakano $^{3}$ - Sanem E. Akarcan ${ }^{5}$ - Necil Kutukculer ${ }^{5}$. Guzide Aksu ${ }^{5}$. \\ Yoji Sasahara ${ }^{3}$. Shigeo Kure ${ }^{3}$. Hidenori Ohnishi ${ }^{4}$. Jean-Laurent Casanova ${ }^{1,2,6,7,8}$ • Anne Puel ${ }^{1,2,6}$ - Toshiyuki Fukao ${ }^{4}$
}

Published online: 4 May 2020

(C) Springer Science+Business Media, LLC, part of Springer Nature 2020

\section{Correction to: Journal of Clinical Immunology (2020)} https://doi.org/10.1007/s10875-020-00770-1

The original version of our manuscript, entitled, “ The ILIRN mutation creating the most-upstream premature stop codon is hypomorphic because of a reinitiation of translation" unfortunately contained mistakes in Fig. 1a and d legends. The text should read as follows:

a. Immunoblot analysis with an anti-FLAG antibody of HEK293 T cells transfected with insert-free FLAG-tagged vector (mock) or with expression vectors carrying various $I L 1 R N$ alleles: WT, R29X, or R29X-M38A (methionine 38 to alanine mutation, blocking reinitiation of translation at M38) or E57X.

d. Protein structure model of IL-1Ra and IL-1R1. IL-1Ra (blue) was superimposed on EBI-005 in complex with IL-1R1 (green).

Publisher's Note Springer Nature remains neutral with regard to jurisdictional claims in published maps and institutional affiliations.

The online version of the original article can be found at https://doi.org/ 10.1007/s10875-020-00770-1

Kunihiko Moriya

kunihiko.moriya.a3@tohoku.ac.jp

Hidenori Ohnishi

ohnishih@gifu-u.ac.jp

1 Laboratory of Human Genetics of Infectious Diseases, Necker Branch, INSERM UMR, 1163 Paris, France

2 Imagine Institute, University of Paris, Paris, France

3 Department of Pediatrics, Tohoku University Graduate School of Medicine, Sendai, Japan
4 Department of Pediatrics, Graduate School of Medicine, Gifu University, Gifu, Japan

5 Department of Pediatric Immunology and Rheumatology, Ege University Faculty of Medicine, Izmir, Turkey

6 St. Giles Laboratory of Human Genetics of Infectious Diseases, Rockefeller Branch, The Rockefeller University, New York, NY, USA

7 Pediatric Hematology-Immunology Unit, Necker Hospital for Sick Children, Paris, France

8 Howard Hughes Medical Institute, New York, NY, USA 